\title{
Pulsar Timing at Urumqi Astronomical Observatory
}

\author{
Na Wang ${ }^{1,2}$, XinJi $W_{u^{2}}{ }^{2}$ Jin Zhang ${ }^{1}$, R. N. Manchester ${ }^{3}$, Aili Yusup ${ }^{1}$, \\ K. S. Cheng ${ }^{4}$ \\ ${ }^{1}$ Urumqi Astronomical Observatory, CAS, 40 South Beijing Road, \\ Urumqi 830011, China \\ ${ }^{2}$ Geophysics Department, Peking University, Beijing 100871, China \\ ${ }^{3}$ Australia Telescope National Facility, CSIRO, P.O. Box 76, Epping \\ NSW 1710, Australia
}

${ }^{4}$ Department of Physics, Hong Kong University, Hong Kong, China

\begin{abstract}
A pulsar timing system has been set up using the 25-m Nanshan telescope of the Urumqi Astronomical Observatory. It uses a dual polarization receiver operating at $18 \mathrm{~cm}$ and a filterbank receiver. The data acquisition system is based on a PC using the Windows NT operating system. Timing properties of about 100 pulsars will be monitored with this system.
\end{abstract}

The Nanshan 25-meter radio telescope, operated by Urumqi Astronomical Observatory of the Chinese Academy of Sciences, is located near the center of Eurasia. The telescope is mainly used as a VLBI station and has receivers for six wavelength bands: $1.3 \mathrm{~cm}, 3.6 / 13 \mathrm{~cm}, 6 \mathrm{~cm}, 18 \mathrm{~cm}$ and $92 \mathrm{~cm}$. It is expected that the telescope will be available for pulsar timing for about two days every week, and so frequent observations will be possible, suitable for monitoring period irregularities such as glitches.

Considering the effects of Galactic background emission, interstellar scattering, dispersion smearing and the spectrum of pulsars, it was decided to use the $18 \mathrm{~cm}$ band for the pulsar timing observations. The telescope has cassegrain optics and uses a horn feed receiving orthogonal circular polarizations. The receiver has dual-channel room-temperature preamplifiers with a system noise of approximately $100 \mathrm{~K}$. After conversion, the signals are fed to a filterbank system which has $1282.5-\mathrm{MHz}$ channels for each polarization. Signals from each channel are then one-bit digitized and recorded by a data acquisition system based on a PC operating under Windows NT. Time and frequency reference signals are obtained from the Observatory $\mathrm{H}$-maser and GPS monitoring system.

The online code is a Windows program written in $\mathrm{C}++. \mathrm{A}$ control card (National Instruments PCT-DIO-96) is used to set the digitizer sampling interval and to control the time synchronization, and a high-speed parallel interface (PCIDIO-32HS) is used for data transfer. A GPIB card is used for time transfer. The data are unpacked and folded synchronously with the pulsar period by the online program. Folded data are saved to disk at every sub-integration time, typically 120 seconds. A sequence counter and a time flag are checked every sample and on each 5 second respectively. For data analysis we use TREDUCE (supported 


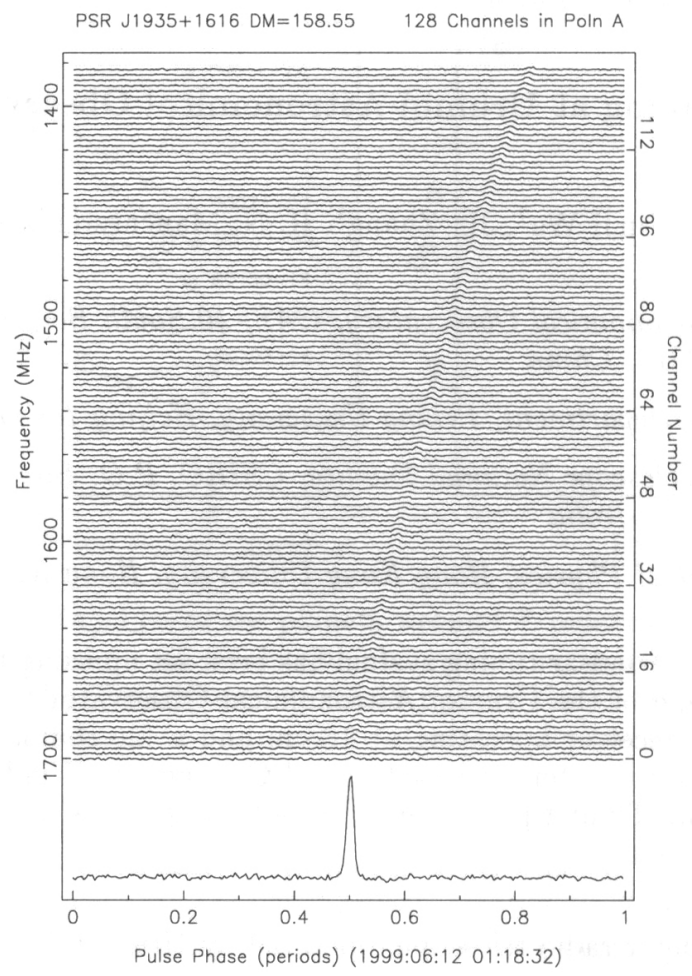

Figure 1. Mean pulse profiles for each channel of one polarization of the filterbank system from a 10-min observation of PSR B1933+16. The dedipersed profile summed over all channels is shown at the bottom.

by Swinburne University of Technology and ATNF) and TEMPO (supported by Princeton University and ATNF) operating under Linux.

First observations were made with the system in mid-1999 (Fig. 1). So far we have detected more than 80 pulsars, with the lowest mean flux density about $4 \mathrm{mJy}$. The project aims to build a more sensitive dual-polarization cryogenic receiver with system temperature of about $30 \mathrm{~K}$ so that pulsars with a mean flux density as low as $1 \mathrm{mJy}$ can be observed.

The most recent published parameters of many pulsars are up to 20 years old. It is worthwhile to reobserve these pulsars to improve their parameters. About 100 pulsars should be detectable using our current system. The timing properties and proper motions of these pulsars will be investigated.

Acknowledgments. The filterbank and digitiser were constructed at the University of Manchester, Jodrell Bank Observatory, and we thank Prof. Andrew Lyne for his support of this project. The down-converter system was constructed at the Australia Telescope National Facility and we thank them for their assistance. 